\title{
PROCESSING AND STRUCTURE OF PAPERBOARD WITH A NEEDLED MIDDLE LAYER
}

\author{
MARIA KLINGBERG, ${ }^{*}$ ANTAL BOLDIZAR ${ }^{*}$ and KARIN HOFER ${ }^{* *}$ \\ *Department of Materials and Manufacturing Technology, Chalmers University of Technology, Gothenburg, \\ Sweden \\ ** Institute of Paper, Pulp and Fiber Technology, Graz University of Technology, Graz, Austria \\ \Corresponding author: Antal Boldizar,antal.boldizar@chalmers.se
}

Received June 6, 2017

\begin{abstract}
The possibility to change the fibre network structure of paperboard by using the needle punching method was studied. Needling was performed on the middle layer made from unbleached softwood kraft pulp at a dry content of $20 \%$. The needled structure was studied by several microscopy methods, which showed that the fibres were redistributed mainly in-plane. Denser regions of the fibre network structure were observed close to the needle penetration sites. The local density was estimated to be $25 \%$ higher than that of the surrounding unaffected structure.
\end{abstract}

Keywords: paperboard, wood cellulose fibre, needle punching, network structure, fibre orientation

\section{INTRODUCTION}

Substantial efforts have been devoted to improve the bending properties of paperboard, such as by modifying the pulp furnish, increasing the distance between denser outer plies or making use of coarser fibres or microspheres in the middle ply. ${ }^{1,2}$ Another approach is to improve the drying process without increasing the bulk density of the paperboard. ${ }^{3}$

Paperboard made by conventional methods is usually described as a porous anisotropic layered structure with fibres primarily oriented in the machine direction, substantially less in the machine cross-direction, and basically no fibres oriented in the paper thickness direction. This anisotropic structure of paper is generally governed by the flow in the machine direction during manufacturing, as most of the fibres are oriented in machine direction, although not perfectly, thus contributing to the cross-direction properties, and very few fibres are tilted in the paper thickness direction. ${ }^{4}$

The possibility to change the two-dimensional fibre structure (2D) towards a three-dimensional structure (3D) has been discussed by many authors, expected to gain a higher compression strength, higher bending stiffness and higher outof-plane strength. The improvement in properties is, however, expected to be at the expense of the in-plane strength. ${ }^{1,5,6}$

The importance of a $3 \mathrm{D}$ fibre structure in paperboard has been pointed out by several authors, as fibres can be tilted into the paper thickness direction by modifying the headbox, adopting high stock consistency methods or by using stiff fibres. An increase in stock consistency was shown to reduce the mobility of the fibres and to reduce the ability of fibres to align with the flow of the fluid, leading to more fibres oriented in different directions and forming a 3D structure. ${ }^{1,6,7}$

In the present study, the possibility to change the fibre network structure by using the needle punching method was explored. The needle punching method is a well-known and commonly used technique in the textile industry since 1870, and it is used in particular to facilitate interlacing of the fibre structure of non-woven multi-layered textiles. ${ }^{8}$ The conventional needle punching method generally results in mechanical bonding of a multilayer textile by rearranging and entangling the fibres, and to some extent also through a reorientation of fibres in the thickness direction. The needling method allows for wide variations in the technique, such as needling from

Cellulose Chem. Technol., 53 (3-4), 315-323(2019) 
one or two sides of the fibre structure, using several needlings in series and making use of different designs of the needle and the barbs.

\section{EXPERIMENTAL} Materials

Unbleached and bleached kraft pulps were used to produce three-ply paperboard. The bleached kraft pulp used for the two outer layers had a composition of approximately $15 \%$ hardwood and $85 \%$ softwood fibres, whereas the middle layer consisted of an unbleached softwood kraft pulp. The average length and thickness of the bleached kraft pulp fibres used were $1.9 \mathrm{~mm}$ and $27 \mu \mathrm{m}$, respectively, and the unbleached fibres had an average length of $2.1 \mathrm{~mm}$ and a thickness of $29 \mu \mathrm{m}$, as determined with a PulpEye device. ${ }^{9}$

All the layers were manufactured using a laboratory handsheet former, type SCAN CM-26:99, giving sheets with an area of about $16 \times 16 \mathrm{~cm}^{2}$ according to EN ISO 5269-1. The volume and concentration of the stock were chosen to obtain mass per unit areas (grammages) of $140 \mathrm{~g} / \mathrm{m}^{2}$ and $100 \mathrm{~g} / \mathrm{m}^{2}$ for the middle and outer layers, respectively. Before the three layers were couched, they were stored with blotting paper between each sheet in sealed plastic bags wrapped in aluminium foil, in order to prevent from drying. During transport to the needling facility at Groz-Beckert $\mathrm{KG}$, the middle layers were kept in boxes with Wettex towels and cooling clamps to keep the moisture content and a low temperature. The reference materials were also transported to Groz-Beckert $\mathrm{KG}$ and exposed to the same temperature and humidity history. In connection to the needling, the samples were stored at a temperature of $7{ }^{\circ} \mathrm{C}$ in order to keep the dry content of the sheets at about $20 \%$.

\section{Needling method}

The needling, performed only on middle layers, was done with a laboratory needle machine type DILOOM OUG-II SB 15 at Groz-Beckert $\mathrm{KG}$ in Germany, at a temperature of $23{ }^{\circ} \mathrm{C}$ and a relative humidity $(\mathrm{RH})$ of $42 \%$. The needle punch machine was equipped with one needle board positioned on the upper side of the loom (Fig. 1). The fibre structure was fed from the inlet and passed between the bed plate and stripper plate. The needles penetrated the material as a result of the vertically oscillating movement of the needle board. The bed plate facilitated the forward movement of the material and the stripper plate prevented the material from following the vertical movement. ${ }^{10}$

The needle board used had 1000 needles of type $15 X 18 X 43 X 3$ R222 G 2017, with a total length of 78 $\mathrm{mm}$ and a $20 \mathrm{~mm}$ working part, as shown in Figure 2. The working part of the needle had an equilateral triangular cross-section with a triangular edge length of $0.40 \mathrm{~mm}$. Each of the three needle edges in the triangular working part of the needle had two barbs, the barbs being of type HL, as designated by Groz-Becker KG. Only the first three barbs (of the total six barbs) closest to the needle tip were used in the present study. The distance between the needle tip and the first barb was $4.8 \mathrm{~mm}$. The barbs along the same edge were 6.36 $\mathrm{mm}$ from each other, whereas the closest distance between the barbs on different edges was $2.12 \mathrm{~mm}$. The direction of the movement in relation to the needling process is described as the needling machine direction (NMD). The other directions are referred to as the needling cross-direction (NCD) and the needling thickness direction (NZD).

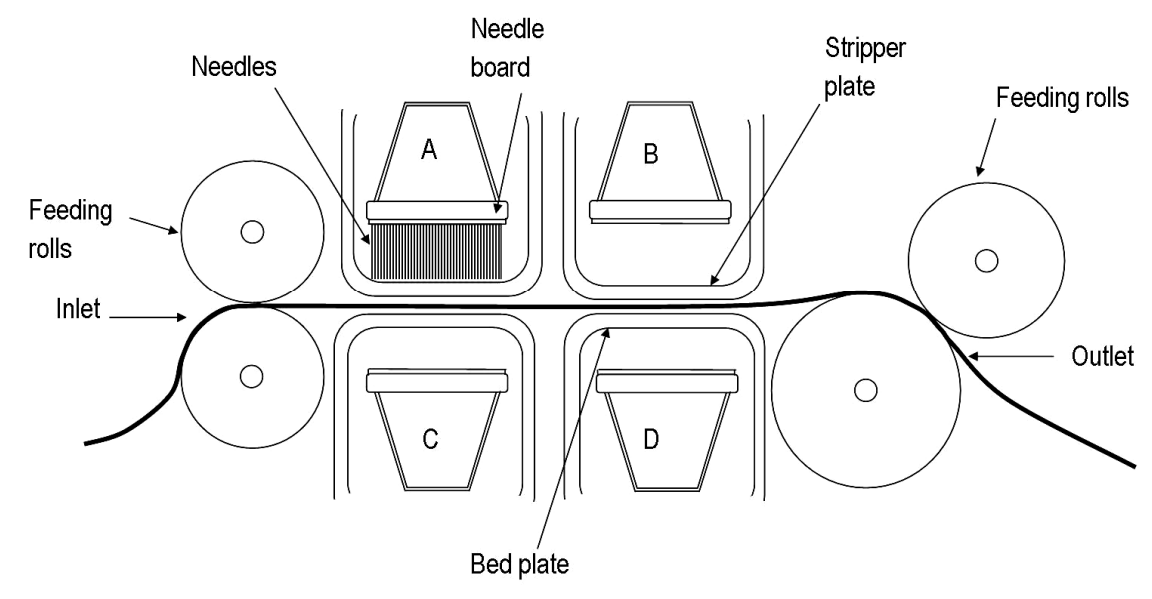

Figure 1: A schematic of the needle punch machine used; the material was fed from the inlet through the needle loom, where the needle punching was performed 
Paperboard

Total length $78 \mathrm{~mm}$

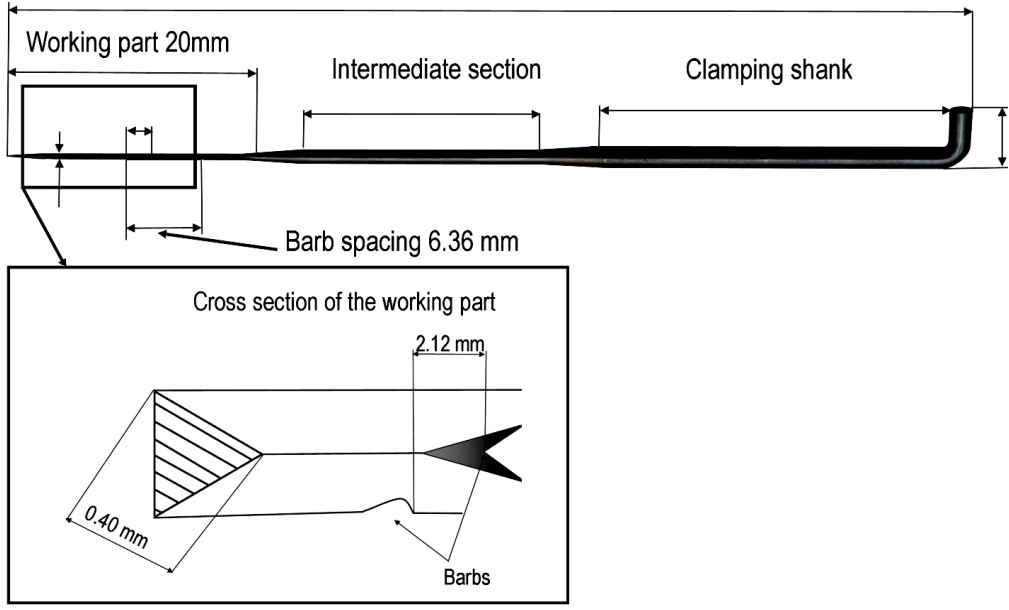

Figure 2: The main geometry of the needle type used (upper part of the figure) and the cross-section of the triangular working part of the needle, with a triangle edge length of $0.40 \mathrm{~mm}$ (lower part of the figure)

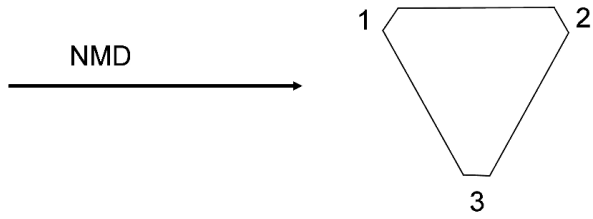

Figure 3: Barbs positioned in relation to the needle machine direction NMD; numbers 1, 2 and 3 refer to numbering of the barbs in ascending order from the needle tip

It is of importance to note that the needle position in relation to the needle machine direction NMD refers to the needled sample and not to the fibre orientation of the paperboard, as shown in Figure 3. Each edge in the figure is marked with a number, describing the barb positioned in relation to the needle tip in ascending order. The first barb (denoted as 1), closest to the needle tip was placed towards the needle machine direction NMD.

Before needling, the middle layers of the board were placed between two greaseproof papers, attached with an adhesive tape to the fabric feeding the sample forward. Needled samples were produced with a needling intensity of 40 stitches $/ \mathrm{cm}^{2}$ and 60 stitches $/ \mathrm{cm}^{2}$, denoted as low and high stitch density, respectively. With needle penetration depths of $4,6,8$ and $10 \mathrm{~mm}$, the number of barbs penetrating the samples was zero, one, two and three barbs, respectively. For both series of low and high stitch densities, the feeding rate was $1 \mathrm{~m} / \mathrm{min}$ at the inlet and $1.15 \mathrm{~m} / \mathrm{min}$ at the exit, resulting in feeding distances per needling stroke of 4.8 and $3.15 \mathrm{~mm}$ for the series of low and high stitch densities, respectively. After the needling, the sheets were transported to Södra Cell, Sweden, where they were couched with outer layers (the outer layers were not needled), dried and conditioned according to EN ISO 5269-1 and EN ISO20187, with the exception that the drying time was extended to 72 hours.

\section{Grammage and thickness measurements}

The samples were conditioned for at least 16 hours at $23{ }^{\circ} \mathrm{C}$ and $50 \% \mathrm{RH}$, before the grammage of the paperboard samples was assessed as the mass per surface area of sheets with trimmed edges. The thickness was measured twice on each subsample with a STFI thickness instrument according to ISO 534:1988 and the average value was calculated. 


\section{Microscopy study of the needled surfaces}

The middle layers, needled with high stitch densities and with zero, one, two and three barbs in penetration, were visually inspected on both sides with a stereomicroscope Stereo Discovery V20, Carl Zeiss. The perforated hole area, as seen on the paper surface, was measured in order to quantify the relative area needled and to estimate the local changes of density in the middle layer. Ten area measurements were made on each sample from the top side and the bottom side at a magnification of 20x. The hole area was measured with the outline function of Axio Vision 4.8 2.0 software.

\section{Microscopy study of the needled cross-section}

The fibre network structure in the cross-section of the needled samples was studied with a Leitz DMRX optical microscope. The viewing direction used is shown in Figure 4. For that purpose, parts of samples approximately $2.5 \times 2 \mathrm{~cm}$ in size were cut and embedded in an acrylic cold moulding compound type DuroCit from Struers A/S. The surface of the embedded sample cross-sections were polished using Silicon Carbide abrasive papers of coarsenesses from P120 to P1000 in six steps, each for 2 min under a load of $10 \mathrm{~N}$.

The samples studied were needled with zero, two and three barbs at a high stitch density and a sample was needled with one barb at low stitch density. The width of the observed denser area close to the hole was measured three times. A total of 18 measurements were performed on each of the four samples.

The average densified widths were converted into densified areas and densified volumes, according to a model assuming circular densified regions. The model was then used for estimations of the local density of needled middle layers.

\section{Model used for estimation of the local fibre structure density}

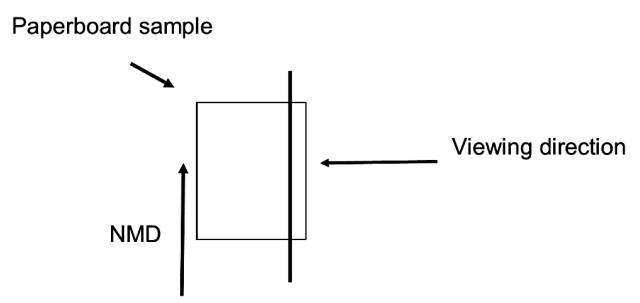

Figure 4: A schematic drawing of the embedded paperboard sample prepared for the microscopy analysis of needled cross-sections (the flat surface sample size was approximately $2.5 \times 2 \mathrm{~cm}$ )
The simplified model used for estimation of the local density of needled middle layers is shown in Figure 5. Here, the perforated hole area (HA) and the perforated hole volume (HV) are related to the densified area (DA) and the densified volume (DV), assuming that the fibres were relocated from $\mathrm{HV}$ to DV by the needling performed. Further assumptions were that the fibres were evenly distributed in all the handmade sheets and that all the fibres from HA were redistributed without any loss of fibres.

The density of the densified volume $\left(\rho_{D V}\right)$ was compared with the surrounding average density of the middle layer $\left(\rho_{G D}\right)$, estimated with Equations 1 and 2, where $\rho_{s}$ and $\rho_{o}$ were the density of the total structure and the density of the outer layers, respectively. The fibre structure outside the local densified regions was considered to be unaffected by the needling.

$$
\begin{aligned}
& \rho_{D V}=\frac{\left(m_{H V}+m_{D V}\right)}{D V} \\
& \rho_{G D}=\frac{\left(t_{s} \cdot \rho_{s}-2 \cdot t_{o} \cdot \rho_{o}\right)}{t_{m}}
\end{aligned}
$$

The mass of the fibres located in the hole volume (HV) before needling is represented with $\boldsymbol{m}_{\boldsymbol{H} V}$ in Equation 3 and the mass of fibres in the densified volume (DV) is represented with $\boldsymbol{m}_{D V}$ in Equation 4, while the thickness of the middle layer $t_{m}$, the total structural thickness $t_{s}$ and the thickness of the outer layers $\boldsymbol{t}_{\boldsymbol{o}}$ were related according to Equation 5.

$$
\begin{aligned}
& m_{H V}=H V \cdot \rho_{G D} \\
& m_{D V}=D V \cdot \rho_{G D} \\
& t_{m}=t_{s}-2 \cdot t_{o}
\end{aligned}
$$

The relative denser area RDA and the relative hole area RHA, quantifying the amount of the middle layer surface area influenced by the needling, were estimated with the needling density $N_{\rho}$ and areas DA and HA with Equations 6 and 7:

$$
\begin{aligned}
& R D A=N_{\rho} \cdot D A \\
& R H A=N_{\rho} \cdot H A
\end{aligned}
$$

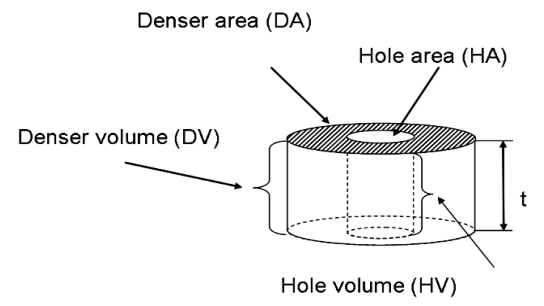

Figure 5: A simplified model of the local structure of the needled middle layer, consisting of a perforated hole area (HA) and a denser area (DA), in the plane surrounded by an average fibre density 
Paperboard
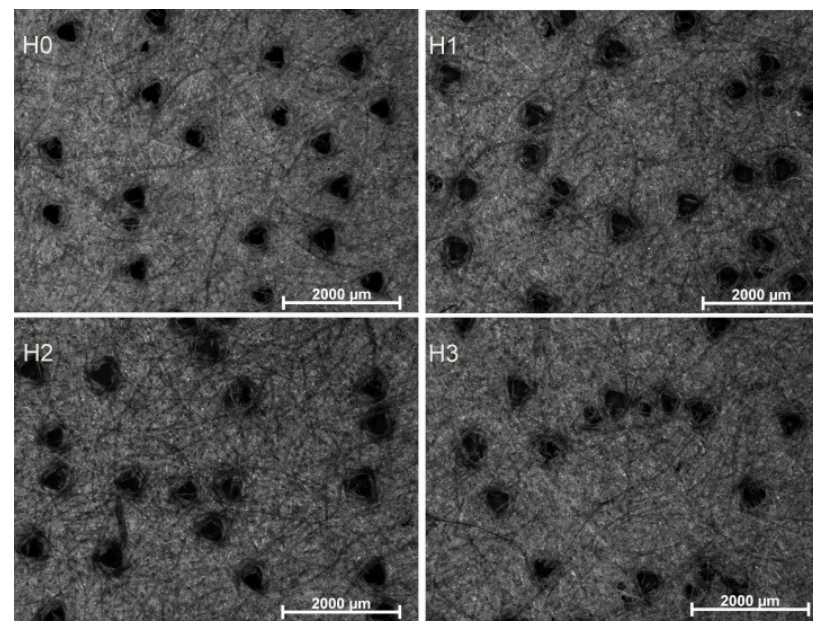

Figure 6: Micrographs showing the top side of single sheets needled at high stitch density with zero, one, two and three barbs, denoted $\mathrm{H} 0, \mathrm{H} 1, \mathrm{H} 2$ and $\mathrm{H} 3$
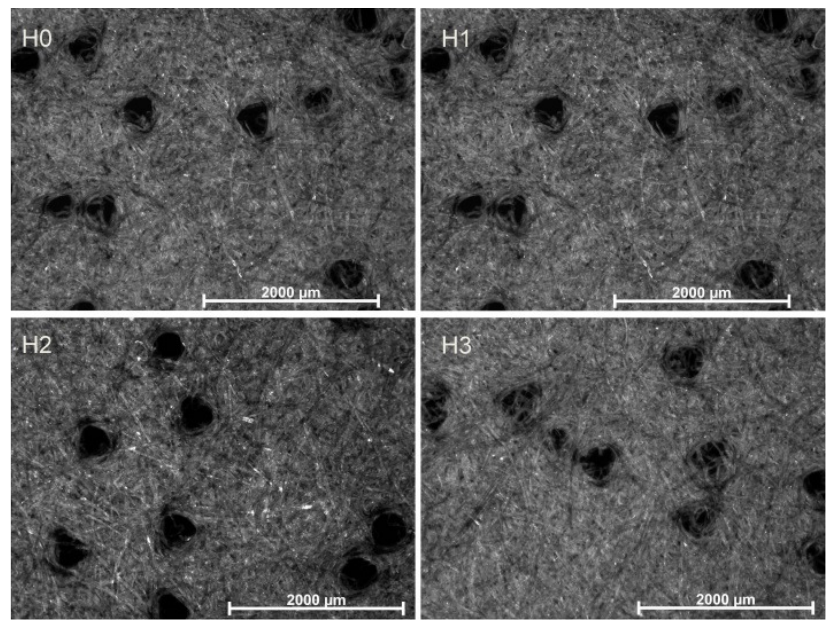

Figure 7: Micrographs of the bottom side of single sheets needled at high stitch density with zero, one, two and three barbs, denoted $\mathrm{H} 0, \mathrm{H} 1, \mathrm{H} 2$ and $\mathrm{H} 3$

\section{D analysis of the local fibre structure}

The fibre structure of the needled middle layers was further studied with a microscopy method based on series of microtomed surfaces, described previously. ${ }^{11,12}$ Here, three-ply paperboard samples with a thickness of 450-500 $\mu \mathrm{m}$ and an area of $8 \times 25$ $\mathrm{mm}$ were prepared with the long side parallel to the NMD. Air was expelled from the fibre network structure by infiltrating the samples for 5 hours with methacrylate-based resin type Technovit 7100, embedded in a gelatine capsule. A rotary microtome with a diamond knife was used to cut $4 \mu \mathrm{m}$ thick slices from the embedded sample. After each third slice, i.e. after $12 \mu \mathrm{m}$, the surface of the sample stub was scanned using a 3D movable light microscope at 20 times magnification, recording single images with a CCD camera. A total of 150 cuts were made on each sample, representing $1800 \mu \mathrm{m}$ in NMD. A 2D stitching of micrographs was used to convert the single images to a composite image of the whole region of interest with an overlap of $10 \% .^{13}$

The region around the needled holes, produced at high needling intensities using zero barb and two barbs, respectively, were studied more closely. One hole in each type of sample is shown, while representative features of two holes in each type of sample are discussed in the results.

\section{RESULTS AND DISCUSSION}

\section{The needled structure}

Figures 6 and 7 show stereo-micrographs, taken from the top side and the bottom side of the needled middle layers of samples produced at high stitch density and penetrated with zero, one, 
two and three barbs. The dark triangular shapes seen are the needled holes. Upon closer examination, it can be seen that the edges of the holes became increasingly rounded for the samples with increasing number of barbs used. In the samples penetrated with one and three barbs, a few fibre ends were visible in the hole area. More disordered fibre ends in the hole area were visible from the bottom side (Fig. 7), especially from three and one barb penetrations.

It was expected that the first barb penetrating the samples would be the most effective in transporting the fibres in the thickness direction, also that increasing the number of barbs acting on the fibre structure would increase the fibre transport and possibly more fibre damage and more round hole edges. ${ }^{14,15}$

No significant differences in hole area could be seen between the different samples produced at high stitch density (Fig. 8). The results indicate, however, that the sample penetrated with no barb had a somewhat smaller standard deviation in HA, which seems reasonable since no barbs had acted on the structure. It was also observed that the two and three barb penetrations caused no significant increase of HA, compared to one barb penetration.

Photomicrographs of the sample cross-sections showed that the middle layer had two regions, referred to as the local denser structure, next to the needled hole, and the surrounding unaffected (average) fibre structure. The denser local structure was seen in all the samples of needled middle layers (Fig. 9). There was no significant difference in the width of the denser regions of the samples, measured to be 122,101 and $97 \mu \mathrm{m}$, with standard deviations of about 30 to $40 \mu \mathrm{m}$, for the samples needled at high stitch density and two, zero and three barbs penetration, respectively.

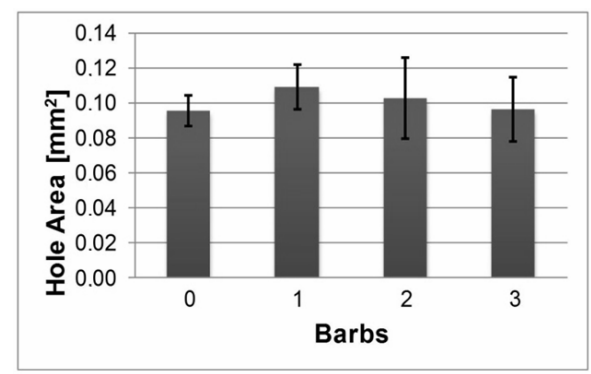

Figure 8: Measured triangular hole area, as seen from the top side of samples needled at high stitch density with zero, one, two and three barbs
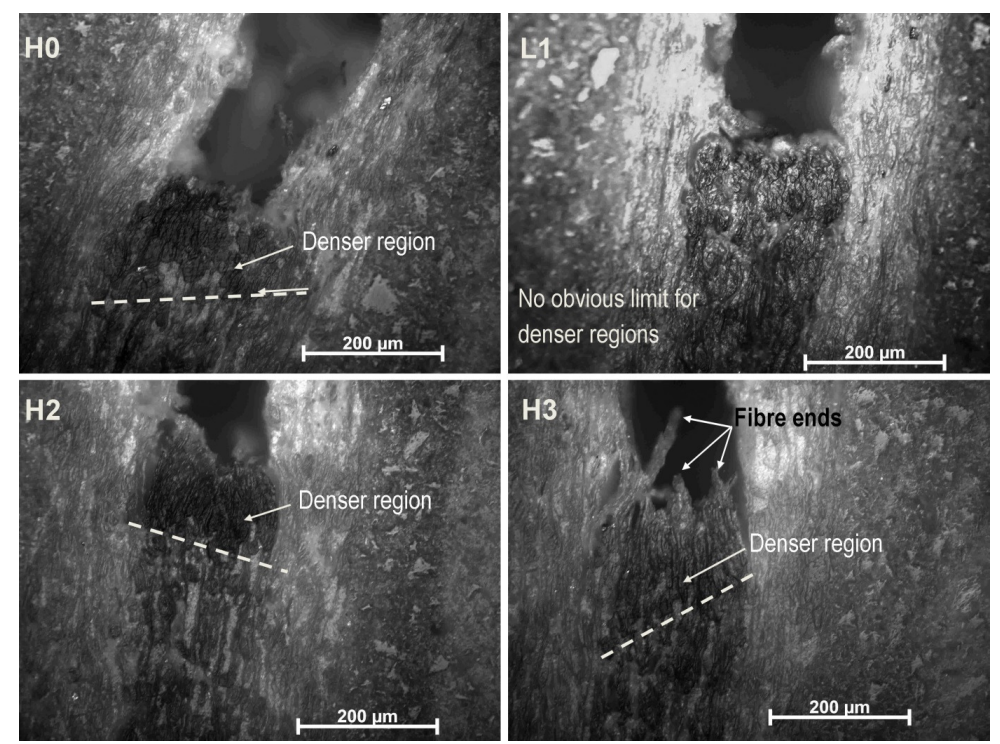

Figure 9: Photomicrographs showing the extent of the local denser regions caused by needling; samples shown were needled at high stitch density $(\mathrm{H})$ and low stitch density $(\mathrm{L})$ with zero, one, two and three barbs, denoted H0, L1, H2 and $\mathrm{H} 3$ 


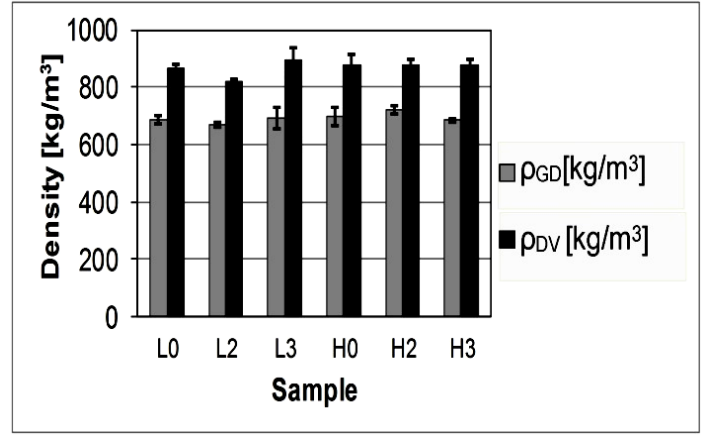

Figure 10: Estimated local density $\rho_{\mathrm{DV}}$ around the needled holes for middle layer samples and the unaffected global density $\rho_{\mathrm{GD}}$, according to Eqs. 1-5 (sample designation as in Fig. 9)

There were no significant differences in the estimated local densities between the samples studied. The main result seen was that the local density in the densified regions was about $25 \%$ higher than the average density in all the needled samples, as shown in Figure 10.

Figure 11 shows the influence of needle stitch density and the number of barbs on the relative extent of the densified area, as estimated with Equations 6 and 7. In the samples needled with low stitch density, about $6 \%$ of the total area of the samples was converted to a denser structure, whereas about $4 \%$ of the global structure was converted to holes. With the high needle stitch density about $9 \%$ of the initial global structure was densified and about $6 \%$ was converted to holes.

\section{Detailed study of the local structure}

A more detailed study of the needled three-ply fibre structure was carried out as a 3D analysis of photomicrographs of the samples having a middle layer needled at high stitch density with zero and two barbs in penetration ( $\mathrm{H} 0$ and $\mathrm{H} 2)$. Four sequences of images are shown in Figure 12, taken along NMD, the position of each sequence indicated in Figure 13. In each sequence, the two samples $\mathrm{H} 0$ and $\mathrm{H} 2$ were compared regarding the local structure of the middle layer. In each image sequence, samples $\mathrm{H} 0$ are on the left-hand side and $\mathrm{H} 2$ on the right-hand side. Each image shows a cross-section of the top layer (upper part), the middle layer and the bottom layer. The NCD is horizontal and the NZD is vertical in the figure,

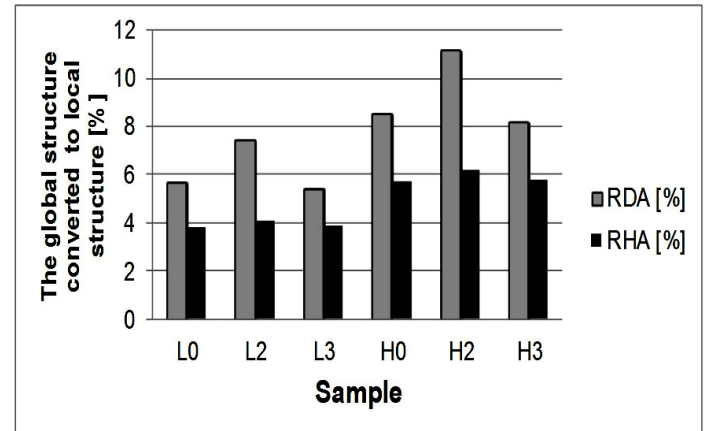

Figure 11: Effect of needle density and number of barbs on the estimated proportion of the area of the original fibre structure converted to a denser local structure and to a perforated hole area, shown as the relative denser area RDA and the relative hole area RHA estimated with Eqs. 6 and 7

while the NMD progresses through sequences $\mathrm{S} 1$ to S4. The sections S1 to S4, representing about $400 \mu \mathrm{m}$ removal of the sample structure, show a hole growing and finally closing along NMD.

The first image S1 in Figure 12 shows the beginning of the hole, close to the leading edge of the needle. At the right-hand side of the hole in both $\mathrm{H} 0$ and $\mathrm{H} 2$, a local darker area is seen. The darker area seems denser in $\mathrm{H} 2$ since less polymer filling can be observed. The darker areas of both $\mathrm{H} 0$ and $\mathrm{H} 2$ probably consist of fibres randomly reoriented in the NMD/NCD plane. On the lefthand side of the penetrated hole, both samples appear to contain fibres oriented according to the structure of the original handsheets before needling, but the structure seems to be somewhat more compressed.

In image S2 in Figure 12, the darker area at the right-hand side of the hole seems to be larger in $\mathrm{H} 0$ than in $\mathrm{H} 2$, indicating some difference between the samples. In H2, a more compressed and smaller width of the densified area can be observed and more fibres are tilted in the NZD close to the hole than in sample H0. The compressed structure may be reasonable, since needling of wet fibre structures can be expected to cause a significant compression of the structure. ${ }^{16}$

In image S3 of Figure 12, the right-hand side next to the needled hole is less dark, but it is still possible to observe some compressed structure on both sides of the hole. In the last image S4 of Figure 12, the hole is seen to be closing and the darker area is shifted to the left-hand side of the hole. The randomly distributed fibre orientation in 
NMD/NCD in both $\mathrm{H} 0$ and $\mathrm{H} 2$ is now on the lefthand side, and the right-hand side exhibits normal fibre orientation.

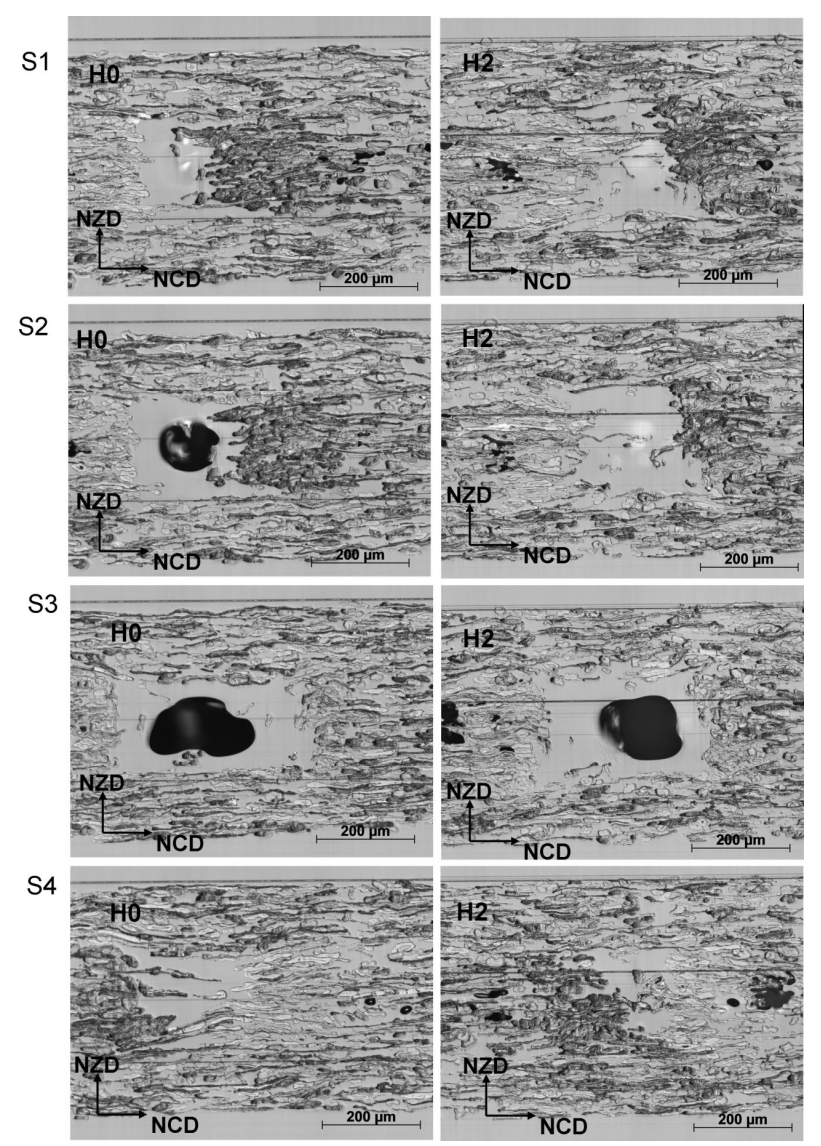

Figure 12: Photomicrographs of three-ply paper board structures of $\mathrm{H} 0$ (left) and $\mathrm{H} 2$ (right), where the sequence S1 to S4 shows the local fibre structure in four positions along the needling machine direction (the positions of S1 to S4 in relation to the needle geometry are indicated in Fig. 13)

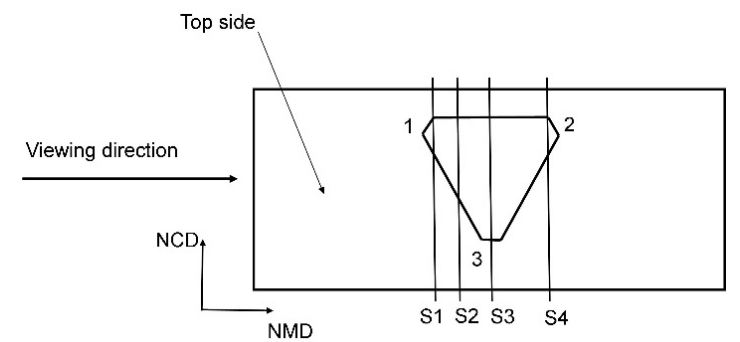

Figure 13: A schematic drawing of the positions of the cross-sections in Fig. 12, relative to the needled hole (the numbering of the needle edges shows the order of the barbs acting on the fibre structure)

\section{CONCLUSION}

It was concluded that the fibres were redistributed in the plane close to the regions of needle penetrations. Micrographs showed no significant differences in the extent of the densified local regions between different types of samples.
The detailed study of the local structure of the sample needled with no barbs and with two barbs indicated a somewhat denser local region and more fibres tilted in the thickness direction for the samples needled with two barbs, but the significance of the differences seen is unclear. 
The needling performed led to an increase in local fibre structure density of approximately $25 \%$. There was no significant difference in the size of the needled holes in the different samples.

Free and disordered fibre ends were observed in samples needled with one and three barbs, indicating a greater fibre damage or fibre pull-out in these samples, where the action of the first barb acting on the samples probably caused most of the free fibre ends seen in the hole regions.

ACKNOWLEDGEMENT: The authors thank the Swedish Government Agency for Innovation Systems (VINNOVA) for financial support. Formulosa, the cooperation between Södra, Tetra Pak and Billerud Korsnäs, is gratefully acknowledged for financial support and for contributing with their expertise. Dr J. A. Bristow is thanked for the linguistic revision.

\section{REFERENCES}

C. Fellers, in "Paper Structure and Properties. The Significance of Structure for Compression Behavior of Paperboard", edited by J. A. Bristow and P. Kolseth, Marcel Dekker Inc., 1986, pp. 281-310.

2 A. J. Svagan, M. A. S. A. Samir and L. A. Berglund, Adv. Mater., 20, 1263 (2008), https://doi.org/10.1002/adma.200701215
3 J. Schlegel, Wochenblatt fuer Papierfabrikation, 135, 614 (2007).

4 N. Stenberg, J. Pulp Pap. Sci., 30, 22 (2004).

5 T. J. Cichoracki, E. Gullichsen and H. Paulapuro, Tappi J., 84, 61 (2001).

6 J. M. Considine, D. W. Vahey and R. Gleisner, Tappi J., 9, 25 (2010).

7 T. Waris, Paper Technol., 31, 14 (1990).

8 B. Attwood and G. Moore, in "An Introduction to the Theory and Practice of Multiply Forming", Pira Technology Series, Edgerton Publishing Services, 1995, pp. 117-120.

9 M. E. Marley, Paper Technol., 49, 23 (2008).

10 I. M. Hutten, in "Handbook of Nonwoven Filter Media”, Butterworth-Heinemann, Oxford, 2007, pp. 195-244.

11 H. Schäffner, PhD Thesis, Graz University of Technology, Austria, 2012.

12 M. Wiltsche, M. Donoser, W. Bauer and $\mathrm{H}$. Bischof, in Procs. 13 ${ }^{\text {th }}$ Fundamental Research Symposium, Cambridge, September, 2005, p. 856.

13 M. Wiltsche, M. Donoser, J. Kritzinger and W. Bauer, J. Microsc., 242, 197 (2011), https://doi.org/10.1111/j.1365-2818.2010.03459.x

14 M. Miao, Text. Res. J., 74, 394 (2004), https://doi.org/10.1177/004051750407400504

15 M. Miao, H. E. Glassey and M. Rastogi, Text. Res. J., 74, $485 \quad$ (2004) https://doi.org/10.1177/004051750407400604

16 S. Debnath and M. Madhusoothanan, J. Ind. Text., 41, $292 \quad$ (2012), https://doi.org/10.1177/1528083711416394 